\title{
ON DUO RINGS
}

\author{
G. Thierrin \\ (received December 17, 1959)
}

Following E. H. Feller [1], a ring $R$ is called a duo ring if every one-sided ideal of $R$ is a two-sided ideal.

In the first part of this paper, we give some properties of duo rings and we show that the set of the nilpotent elements of a duo ring $R$ is an ideal, the intersection of the completely prime ideals of $R$.

It is easy to see that every duo ring is a subdirect sum of subdirectly irreducible duo rings. We give in the second part of this paper a characterization of the subdirectly irreducible duo rings. This characterization is quite similar to the characterization of the subdirectly irreducible commutative rings, due to N.H. McCoy [2], whose methods we use.

1. Prime ideals in the duo rings. If $R$ is a duo ring, one sees easily that for every triple of elements, $a, b, c \in R$, there exist $x, y \in R$ such that

$$
a b c=b x=y b .
$$

PROPOSITION 1. Every idempotent element e of a duo ring $R$ is central.

Proof. If $a \in R$, the re exist $x, y \in R$ such that aee $=e x$ and eea $=$ ye. "Hence eae $=e x=y e$ and $a e=e a$.

PROPOSITION 2. Every non-nilpotent minimal ideal $M$ of a duo ring $R$ is a division ring.

Can. Math. Bull. vol. 3, no. 2, May 1960 
Proof. Because $R$ is a duo ring, $M$ is also a minimal right ideal. Therefore, there exists an idempotent element e such that $M=e R$. Since $e$ is central by proposition 1 , e is an identity element of $M$. If $m \in M, m \neq 0$, we have $m M \neq 0$; hence $m M=M$ and $M$ is a division ring.

THEOREM 1. Every duo ring R (of more than one element), which is subdirectly irreducible and without non-zero nilpotent elements, is a division ring.

Proof. Since $R$ is subdirectly irreducible, it contains a minimal ideal $\mathrm{M}$, the intersection of the non-zero ideals of R. This ideal $M$ is not nilpotent. Therefore, by proposition 2, $M$ is a division ring and there exists a central idempotent element e such that $M=e R$. The set $T=\{e x-x \mid x \in R\}$ is an ideal of $R$. If $y \in M \cap T$, we have $y=e y=0$. Hence $M \cap T=0$ and $T=0$. Therefore $e x=x$ for every $x \in R$ and $M=R$.

An ideal $P$ of a ring $R$ is said to be prime, if $X Y \subseteq P$ implies that $X \subseteq P$ or $Y \subseteq P, X$ and $Y$ being ideals of $R$. According to McCoy [3], an ideal $P$ is prime if and only if $x R y \subseteq P$ implies that $x \in P$ or $y \in P$. An ideal $Q$ of $R$ is said to be completely prime, if $x y \in Q$ implies that $x \in Q$ or $y \in Q$.

PROPOSITION 3. Every prime ideal $P$ of a duo ring $R$ is completely prime.

Proof. Let $x y \in P$. The set $T=\{t \mid t \in R$, $x t \in P\}$ is a right ideal, the refore a two-sided ideal of $R$. As $y \in T$, we have $R y \subseteq T$ and $x R y \subseteq P$. Hence $x \in P$ or $y \in P$.

THEOREM 2. The set $\mathrm{N}$ of the nilpotent elements of a duo ring is an ideal, which is the intersection of the completely prime ideals of $R$.

Proof. Let I be the intersection of the completely prime ideals $P_{i}$ of $R$. If $a^{n}=0$, we have $a^{n} \in P_{i}$ and therefore $a \in P_{i}$. Hence $N \subseteq I$. From the proposition 3 , it follows that $I$ is the intersection of the prime ideals of $R$. Now, according to McCoy [3], this intersection is a nil ideal. Therefore $\mathrm{N}=\mathrm{I}$.

As any homomorphic image of a duo ring is also a duo ring, we have the following: 
COROLLARY. Every duo ring without non-zero nilpotent elements is a subdirect sum of duo rings without divisors of zero.

2. Subdirectly irreducible duo rings. As every ring is a subdirect sum of subdirectly irreducible rings and every homomorphic image of a duo ring is a duo ring, we have the following.

THEOREM 3. Every duo ring is a subdirect sum of subdirectly irreducible duo rings,

We shall now characterize the subdirectly irreducible duo rings. To do this, it will suffice to adapt the arguments used by McCoy in [2] to characterize the subdirectly irreducible commutative rings. As in [2], we shall distinguish two cases.

Case 1. In this case, we consider rings, not all of whose elements are right divisors of zero. We shall now prove:

THEOREM 4. Let $\mathrm{R}$ be a duo ring with at least one element which is not a right divisor of zero, and let $D$ be the set of all right divisors of zero in $R$. Then $R$ is subdirectly irreducible if, and only if, it has the following four properties:

(1) The set of all elements $x$ of $R$ such that $x D=0$ is a principal ideal $\mathrm{J}=(j) \neq 0$.

(2) The set of all elements y of $R$ such that $\mathrm{Jy}=0$ is precisely $D$. (Hence $D$ is an ideal in $R$.)

(3) $R / D$ is a division ring.

(4) If $d$ is any element of $D$ which is not in $J$, there exists an element $c$ of $R$ such that $d c=j$.

Proof. By theorem 1, if $\mathrm{R}$ has no non-zero nilpotent elements and is subdirectly irreducible, $R$ is a division ring and hence the above properties are trivially satisfied. Conversely, if $R$ has the above stated properties with $D \neq 0$, then $R$ has a non-zero nilpotent element. Hence, if $R$ has these properties and contains no non-zero nilpotent elements, $D=0$ and $R$ is a division ring. Accordingly, we may henceforth confine our attention to the case in which $R$ has at least one non-zero nilpotent element. 
We shall first show that if $R$ has the stated properties, it is subdirectly irreducible. To show this, we shall show that every principal ideal (a), a $\neq 0$, contains $\mathrm{J}$.

First, let a be any non-zero element of $J$. Since $R$ is a duo ring, we have

$$
a=j b+n j \neq 0 \quad(b \in R, n \text { an intege } r) .
$$

Let $c$ be an element of $R$ which is not a right divisor of zero. Then

$$
a c=(j b+n j) c=j(b c+n c) \neq 0 .
$$

Hence $b c+n c$ is not a right divisor of zero. Thus, by (3), there exists an element $x$ of $R$ such that

$$
(\mathrm{bc}+\mathrm{nc}) \mathrm{xc}=\mathrm{c}+\mathrm{d}
$$

where $d$ is an element of $D$. Multiplying by $j$, we get

$$
j(b c+n c) x c=j c+j d=j c .
$$

Thus, since $c$ is not a right divisor of zero, we have

$$
j=j(b c+n c) x=(j b+n j) c x=a c x .
$$

Therefore $j \in(a)$ and $J \subseteq(a)$.

If $a$ is an element of $D$, not in $J$, then (a) contains $J$ by property (4).

If, finally, a is not a right divisor of zero, by (3) there exists an element $x$ such that

$$
\text { axa }=a+d \quad(d \in D) \text {. }
$$

Hence, $j a x a=j a$ and $j a x=j$. Therefore $j \in(a)$ and $J \subseteq(a)$. We have thus established this part of the theorem.

We assume henceforth that $R$ has at least one non-zero nilpotent element and is subdirectly irreducible. Let $\mathrm{J}$ be the unique minimal ideal of $R$. Clearly $J$ is a principal ideal and is generated by any of its elements other than the zero element. We let $j$ be any fixed non-zero element of $J$, so that $J=(j)$.

If $a$ is any non-zero element of $R, a R$ is a non-zero ideal in $R$ and hence contains $J$. Thus, there exists an element $x$ of $R$ such that

$(\alpha)$

$a x=j$ 
By theorem 2, the set $N$ of the nilpotent elements of $R$ is an ideal. Hence $\mathrm{J} \subseteq \mathrm{N}$ and $\mathrm{j}$ is nilpotent. If $\mathrm{j}^{2} \neq 0$, there exists by $(\alpha)$ an element y such that $j^{2} y=j$. This, however, is seen to be inconsistent with the nilpotence of $j$. Hence we must have $j^{2}=0$.

Proof of (2). If $\mathrm{Jy}=0$, then $y \in D$. Conversely, if $d \in D$, there exists $z \neq 0$ such that $z d=0$. The set $\{t \mid t \in R, t d=0\}$ is a non-zero ideal of $R$ and therefore contains $J$. Hence $J d=0$ and $\mathrm{D}$ is an ideal.

Proof of (3). If $c$ is any element which is not a right divisor of zero, the ideal $j c R$ is a non-zero ideal, since $j c^{2} \neq 0$. Hence $J \subseteq j c R$ and there exists $x \in R$ such that $j=j c x$. If $a$ is an a rbitrary element of $R$, we have $j a=j c x a$ and $j(a-c x a)=0$. Hence, a - cxa is a right divisor of zero and $a-c x a \in D$. Therefore, $R / D$ is a division ring.

Proof of ( 1$)$. Let a be any non-zero element of $R$ such that $a D=0$. By $(\alpha)$, there exists $x$ such that $a x=j$. If $c \notin D$, we have axc $=j c \neq 0$ and $x c \notin D$. Hence, by (3), there is an element $t \in R, t \notin D$, such that

$$
x c t=c+d \quad(d \in D) \text {. }
$$

Hence

$$
j c t=a x c t=a(c+d)=a c .
$$

Since $c, t \& D$, there exists by (3) an element $v$ such that

$$
c t=v c+d_{1} \quad\left(d_{1} \in D\right) \text {. }
$$

Hence $j c t=j v c+j d_{1}=j v c$. Therefore, $j v c=a c, j v=a$ and $a \in J$.

Proof of (4). This is immediate by $(\alpha)$.

Case 2. In this case, we consider rings, all of whose elements are right divisors of zero. We have the following.

THEOREM 5. Let $\mathrm{R}$ be a duo ring in which all elements are right divisors of zero. Then $R$ is subdirectly irreducible if, and only if, it has the following three properties:

(1) There exists a fixed prime $p$ such that if $a R=0$, then $\mathrm{p}_{\mathrm{a}}=0$ for some positive integer $\mathrm{k}$, depending on $\mathrm{a}$. 
(2) The set of all elements a of $R$ such that $a R=0, p a=0$, is a principal ideal $\mathrm{J}=(\mathrm{j}) \neq 0$.

(3) If $b R \neq 0$, the re exists an element $c$ such that $b c=j$.

The proof of this theorem is identical to the proof of the corresponding theorem in [2].

\section{REFERENCES}

1. E. H. Feller, Properties of primary noncommutative rings, Trans. Amer. Math. Soc. 89 (1958), 79-91.

2. N. H. McCoy, Subdirectly irreducible commutative rings, Duke Math. J. 12 (1945), 381-387.

3. N. H. McCoy, Prime ideals in general rings, Amer. J. Math. 71 (1949), 823-833.

University of Montreal

and

Summer Research Institute, Kingston 